Pacific Journal of Mathematic 


\section{GENERALIZED CONVOLUTIONS AND POSITIVE DEFINITE FUNCTIONS ASSOCIATED WITH GENERAL ORTHOGONAL SERIES}

\section{ALAN SCHWARTZ}

Let $\left\{\phi_{n}\right\}$ be a sequence of continuous functions orthogonal on an interval with respect to a positive measure $d \alpha$, and let $h(n)=\left(\int_{a}^{b}\left|\phi_{n}\right|^{2} d \alpha\right)^{-1}$. Then under hypotheses general enough to include as special cases the trigonometric system $\left\{e^{i n x}\right\}$, the ultraspherical polynomials, and most cases of the Jacobi polynomials, the sequences $\langle a\rangle$ satisfying $\|a\|=\sum_{n=0}^{\infty}|a(n)| h(n)<$ $\infty$ form a Banach algebra with a convolution defined by $\langle a * b\rangle=$ $\langle c\rangle$ where $\sum_{n=0}^{\infty} c(n) h(n) \phi_{n}=\left(\sum_{n=0}^{\infty} a(n) h(n) \phi_{n}\right)\left(\sum_{n=0}^{\infty} b(n) h(n) \phi_{n}\right)$. Attention is centered upon sequences $\langle a\rangle$ of unit norm (called distribution sequences), and the associated orthogonal series $\sum a(n) h(n) \phi_{n}$ (called characteristic functions). Theorems on divisibility and stability of these classes are proved, the results being modeled after the corresponding ones about the class of characteristic functions in probability theory.

In $\S 1$ the classical example and the one corresponding to the ultraspherical polynomials are discussed. Section 2 is devoted to the study of the basic properties of characteristic functions. The principle results of this section are Theorem 1 (c) which relates convergence of distribution sequences and characteristic functions and Theorem 2 (f) which gives a canonical form analogous to that of Lévy and Khinchine for the divisible characteristic functions, i.e., those characteristic functions which have roots of every order in the class of characteristic functions. An important collection of semigroups of characteristic functions (the stable ones) is classified in $\S \S 3$ and 4 . Section 5 contains several examples. An excellent exposition of the classical case can be found in Lukac's book [12]; this paper makes heavy use of generalizations of techniques which are to be found there.

The author wishes to acknowledge his debt of gratitude to Richard Askey for suggesting the problems that are solved here, and for encouragement throughout this investigation.

1. Examples. Recall the definition and elementary properties of characteristic functions in the classical case:

A distribution function $F$ is a nondecreasing function defined on $(-\infty,+\infty)$ such that $F(x) \rightarrow 0$ as $x \rightarrow-\infty$ and $F(x) \rightarrow 1$ as $x \rightarrow+\infty$. A real-valued random variable $X$ has distribution $F$ if $P[X \leqq x]=$ $F(x)$. The characteristic function of $F$ is its Fourier-Stieltjes trans- 
form:

$$
f(t)=\int_{-\infty}^{\infty} e^{i t x} d F(x) .
$$

If $X$ and $Y$ are independent real-valued random variables with distributions $F$ and $G$, and if $f$ and $g$ are the characteristic functions of $F$ and $G$ respectively, then $X+Y$ has distribution $H=F * G$, the convolution of $F$ and $G$, where

$$
F * G(x)=\int_{-\infty}^{\infty} F(x-y) d G(y),
$$

and the characteristic function $h$ of $H$ is given by $h=f g$. Thus, questions about convolutions of distributions can be recast as questions about products of characteristic functions. Much of the work in this paper will correspond more closely to the situation that arises in those cases where the random variables are integer-valued. In that case, the distribution can be thought of as a sequence $u(n)=P[X=n]$, with $u(n) \geqq 0$ and $\sum_{n=-\infty}^{\infty} u(n)=1$. The characteristic function becomes the series

$$
f(x)=\sum_{n=-\infty}^{\infty} u(n) e^{i n x}
$$

and the convolution $\langle w\rangle$ of distribution sequences $\langle u\rangle$ and $\langle v\rangle$ is given by

$$
w(n)=\sum_{k=-\infty}^{\infty} u(n-k) v(k) .
$$

Similar techniques have been devised to deal with random variables which take values in spaces other than the real line. For instance, $X$ may take values in the unit sphere $\sum_{k-1}$ in $k$-dimensional Euclidean space. If $X$ and $Y$ are random variables in $\sum_{k-1}$, write $X=T p$ and $Y=S p$ where $T$ and $S$ are random variables in $\mathrm{SO}(k)$, and $p=$ $(0,0, \cdots, 0,1)$, and define $X \oplus Y=T S p$. This relation can be used to define a convolution for measure on $\mathrm{SO}(k)$ in such a way that the distribution of TS is the convolution of the distributions of $T$ and $S$. Restriction to random variables satisfying $P[X \in E]=P[X \in T E]$ for every $T \in \mathrm{SO}(k)$ satisfying $T p=p$, yields a theory which takes place on $[-1,1]$ by identification of $X$ with $X \cdot p$. This gives rise to a convolution algebra of measures on $[-1,1]$. The idea analogous to characteristic function here will be a sequence of Fourier-Stieltjes coefficients with respect to a certain orthogonal sequence. More specifically, put $2 \lambda=k-2$, and let $\left\{W_{n}^{\lambda}(x)\right\}$ be the ultraspherical or Gegenbauer polynomials normalized so that $W_{n}^{\lambda}(1)=1$. These polynomials are orthogonal on $[-1,1]$ with respect to the measure 
$\left(1-x^{2}\right)^{\lambda-1 / 2} d x ;\left\{W_{n}^{1 / 2}(x)\right\}$ are the Legendre polynomials. Define

$$
[w(n)]^{-1}=\int_{-1}^{1}\left[W_{n}^{\lambda}(x)\right]^{2}\left(1-x^{2}\right)^{\lambda-1 / 2} d x
$$

If $\mu$ is a measure on $[-1,1]$, and $N=\{0,1,2, \cdots\}$, put

$$
a(n)=\int_{-1}^{1} W_{n}^{\lambda}(x) d \mu(x)
$$

equivalent to the statement

$$
\mu \sim \Sigma a(n) w(n) W_{n}^{\lambda}
$$

Then if

$$
\eta \sim \Sigma b(n) w(n) W_{n}^{\lambda}
$$

and if $\mu^{*} \eta$ is the convolution of $\mu$ and $\eta$,

$$
\mu^{*} \eta \sim \Sigma a(n) b(n) w(n) W_{n}^{\lambda} \quad(n \in N) .
$$

The structure of the algebra and the convolution are discussed in more detail by Bochner [3], Conte [5], and Hirschman [9]. Investigations of stochastic processes associated with this structure can be found in Bochner [3], Roberts and Ursell [13], Lamperti [11], and Bingham [1].

Hirschman [9] pointed out a duality between $n$ and $x$ in $\left\{W_{n}^{\lambda}(x)\right\}$ A distribution sequence is a sequence $\langle a\rangle=\{a(n)\}_{n=0}^{\infty}$ satisfying $a(n) \geqq 0$ and $\Sigma a(n) w(n)=1$; call $f(x)=\Sigma a(n) w(n) W_{n}^{\lambda}(x)(|x| \leqq 1)$ the characteristic function of $\langle a\rangle$. Hirschman [9] describes a dual convolution structure on the distribution sequences. That is, there are nonnegative coefficients $c(k, n, m)$ such that if $\langle a\rangle$ and $\langle b\rangle$ are distribution sequences with characteristic functions $f$ and $g$, and if

$$
c(k)=\Sigma c(k, n, m) a(n) w(n) b(m) w(m) \quad(n, m \in N),
$$

then $\langle c\rangle$ is a distribution sequence with $f g$ as its characteristic function. Thus, there is a well-defined dual to the structure associated with zonal probability distributions on spheres. Problems in this context have been studied by Kennedy [10], and Bingham [2]. Both structures are well defined even if $2 \lambda$ is not an integer.

In this paper, a generalization of this dual structure to a wide class of orthogonal expansions which include Fourier series, ultraspherical series, and certain Jacobi series is formulated.

\section{Characteristic functions.}

Definitions And Notation. Let $I=[a, b]$ be a fixed bounded 
interval; an integral sign without limits will always denote integration over $I$. Let $N$ be the set of nonnegative integers and $N^{\prime}=N-\{0\}$; $\Sigma$ and $\Sigma^{\prime}$ will denote summation over $N$ and $N^{\prime}$ respectively with respect to repeated indices. Let $\alpha$ be a strictly increasing function on $I$, and let $\left\{\phi_{n}\right\}_{n \in N}$ be a sequence of continuous functions on $I$ such that $\int \phi_{n} \bar{\phi}_{m} d \alpha=0$ if $n \neq m$.

Define

$$
h(n)=\left\{\int\left|\phi_{n}\right|^{2} d \alpha\right\}^{-1} \text { and } \quad c(k, n, m)=\int \bar{\phi}_{k} \dot{\phi}_{n} \phi_{m} d \alpha \quad(k, n, m \in N),
$$

and make the following assumptions.

A1. $\left\{\phi_{n}\right\}_{n \in N}$ is a maximal orthogonal set in $L^{2}(d \alpha)$.

A2. $\phi_{0} \equiv 1$.

A3. $\phi_{n}(b)=1(n \in N)$.

A4. For some constant $M$

$$
\left|\phi_{n}(x)\right| \leqq M \quad(n \in N, x \in I) .
$$

$\begin{array}{lll}\text { A5. } & c(k, n, m) \geqq 0 & (k, n, m \in N), \\ \text { A6. } & \Sigma h(k) c(k, n, m)<\infty & (n, m \in N) . \\ \text { A7. } \quad \int \dot{\phi}_{n}^{2} d \alpha \geqq 0 & (n \in N),\end{array}$

REMARKS. Axiom A2 can be replaced by A2': $\int \phi_{n} d \alpha \geqq 0(n \in N)$ and $\Sigma h(n) \int \phi_{n} d \alpha<\infty$. The purpose of either axiom will be to ensure that the unit constant function will be a characteristic function, but $\mathrm{A} 2$ ' adds complications to many otherwise simple arguments. Axiom A7, which is obviously true in the real case, guarantees that the class of characteristic functions will be closed under complex conjugation. A corollary later in the paper will show that the $M$ in A4 can be replaced by unity.

EXAMPLES 1. Exponential functions. Let $a=0, b=2 \pi$, and $d \alpha(x)=d x$; replacing $N$ by $Z=N \cup\left(-N^{\prime}\right)$, let $\phi_{n}(x)=e^{i n x}(n \in Z)$. Then $h(k)=(2 \pi)^{-1}$ and

$$
c(k, n, m)= \begin{cases}2 \pi & k=n+m \\ 0 & \text { otherwise }\end{cases}
$$

2. Orthogonal polynomials. Let $\left\{p_{n}\right\}_{n \in N}$ be a family of real polynomials on $[a, b]$ orthogonal with respect to $d \alpha$ such that the degree of $p_{n}$ is exactly $n$, and such that for some real positive number $M$ 


$$
\left|p_{n}(x)\right| \leqq M p_{n}(b) \quad(x \in I \text { and } n \in N)
$$

and

$$
\int p_{k} p_{n} p_{m} d \alpha \geqq 0 \quad(k, n, m \in N) .
$$

Define $\phi_{n}=p_{n} / p_{n}(b)$, then $\left\{\phi_{n}\right\}_{n \in N}$ satisfies A1-A7: A1, A3, and A4 are obvious. A2 follows because $\phi_{0}=1$, A5 holds because of (1) and (2), and $\mathrm{A} 6$ follows from the fact that $c(k, n, m)=0$ if $k>n+m$. An especially important example of orthogonal polynomials is

3. Jacobi polynomials. Let $a=-1, b=1, d \alpha(x)=(1-x)^{\alpha}(1+x)^{\beta} d x$ and $\phi_{n}=P_{n}^{(\alpha, \beta)} / P_{n}^{(\alpha, \beta)}(1)$ where the $P_{n}^{(\alpha, \beta)}$ are the Jacobi polynomials. Then (1) holds with $M=1$ if $\alpha \geqq \beta$ and $\alpha \geqq-1 / 2[14$, pp. 58 and 168], and (2) holds if $\alpha \geqq \beta$ and $\alpha+\beta+1 \geqq 0$ [6] (actually A5 holds in a slightly larger region [7]). Thus $\left\{\phi_{n}\right\}_{n \in N}$ satisfy A1-A6 if $\alpha \geqq \beta$ and $\alpha+\beta+1 \geqq 0$. Hence the axioms hold for the normalized ultraspherical polynomials $\left\{W_{n}^{i}\right\}$; and, in particular, for Legendre polynomials.

The following lemma will be referred to later.

LemMa 1. Suppose $\int|f|^{2} d \alpha<\infty$ and $\Sigma|u(n)| h(n)<\infty$, where $u(n)=\int f \bar{\phi}_{n} d \alpha$. Then $f=\Sigma u(n) h(n) \phi_{n}$ almost everywhere with respect to $\alpha$. If $f$ is continuous, the series converges to $f$ everywhere.

Proof. $\quad \Sigma u(n) h(n) \dot{\phi}_{n}$ converges uniformly to a continuous function $g$ by the Weirstrass $M$-test. By A4, $\left\{h(n)^{-1}\right\}$ is a bounded sequence so $u(n) \rightarrow 0$ as $n \rightarrow \infty$, hence $\Sigma\left(|u(n)| h(n)^{1 / 2}\right)^{2}<\infty$. Since $\left\{h(n)^{1 / 2} \phi_{n}\right\}$ is a complete orthonormal system with respect to $d \alpha$

$$
\int\left|f-\sum_{n=0}^{k} u(n) h(n) \phi_{n}\right|^{2} d \alpha \longrightarrow 0 \text { as } k \longrightarrow \infty \text {. }
$$

Hence $f=g$ almost everywhere with respect to $\alpha$.

An immediate consequence of the lemma and $\mathrm{A} 6$ is

$$
\dot{\phi}_{n} \dot{\phi}_{m}=\Sigma c(k, n, m) h(k) \dot{\phi}_{k},
$$

evaluating (3) at $b$ yields

$$
\Sigma c(k, n, m) h(k)=1 .
$$

A sequence $\langle u\rangle=\{u(n)\}_{n \in N}$ is called a distribution sequence if $u(n) \geqq 0$ and $\Sigma u(n) h(n)=1$. The continuous function

$$
f=\Sigma u(n) h(n) \phi_{n}
$$


will be called the characteristic function of $\langle u\rangle$. If $\langle u\rangle$ and $\langle v\rangle$ are distribution sequences, define $\langle w\rangle=\langle u * v\rangle$ by

$$
w(k)=\Sigma c(k, n, m) u(m) h(m) v(n) h(n) .
$$

Then from (4)

$$
\Sigma w(k) h(k)=[\Sigma u(m) h(m)][\Sigma v(n) h(n)]=1,
$$

and from $\mathrm{A} 5, w(k) \geqq 0$, so $\langle w\rangle$ is also a distribution sequence. Moreover, the characteristic function of $\langle w\rangle$ is the product of the characteristic functions of $\langle u\rangle$ and $\langle v\rangle$ because of (3).

The following theorem lists some of the useful properties of characteristic functions.

THEOREM 1. (a) Two distribution sequences are the same if and only if their characteristic functions agree.

(b) Convex combinations and products of characteristic functions are also characteristic functions.

(c) Let $\left\{\left\langle u_{j}\right\rangle\right\}_{j \in N^{\prime}}$ be distribution sequences with characteristic functions $\left\{f_{j}\right\}_{j \in N^{\prime}}$. If $\left\langle u_{j}\right\rangle$ converges pointwise to a distribution sequence $\left\langle u_{0}\right\rangle$ with characteristic function $f_{0}$, then $f_{j} \rightarrow f_{0}$ uniformly; conversely, if $f_{j}$ converges pointwise to a function $f$ which is continuous at $b$, then $\left\langle u_{j}\right\rangle$ converges pointwise to a distribution sequence $\langle u\rangle$, the characteristic function of which is $f$, and $f_{j} \rightarrow f$ uniformly.

REMARK. Corresponding to every fact about characteristic functions is one about distribution sequences; for instance, (b) also says that the convolution of distribution sequences is also a distribution sequence. This leads to a kind of stochastic process on the nonnegative integers. We say $X$ is sampled from distribution $v$ if $\operatorname{Pr}[X=k]=$ $v(k) h(k)$. Let $X$ and $Y$ be sampled from distributions $\langle u\rangle$ and $\langle v\rangle$ respectively. Then if it is observed that $X=m$ and $Y=n$, sample $Z$ from the distribution $w(k)=c(k, n, m) h(k)$. Then the distribution of $Z$ is $\langle u * v\rangle$. (This interpretation was suggested by the referee.)

Proof. (a) follows from A1, the first part of (b) is trivial, and the second part follows from the discussion above.

To prove the first part of (c), let $\left\langle u_{j}\right\rangle$ and $f_{j}$ be as in the statement of the theorem, and let $M$ be as in A4. Choose $\varepsilon>0$; since $\left\langle u_{0}\right\rangle$ is a distribution sequence, there exists an integer $K$ such that

$$
\sum_{n=K+1}^{\infty} u_{0}(n) h(n)<\varepsilon / 4 M,
$$

and since $\left\langle u_{j}\right\rangle \rightarrow\left\langle u_{0}\right\rangle$ pointwise, there is an integer $J$ such that if $j \geqq J$ 


$$
\sum_{n=0}^{K}\left|u_{j}(n)-u_{0}(n)\right| h(n)<\varepsilon / 4 M .
$$

Then, if $j \geqq J$

$$
\begin{aligned}
\left|f_{j}(x)-f_{0}(x)\right|= & \left|\sum\left[u_{j}(n)-u_{0}(n)\right] h(n) \phi_{n}(x)\right| \\
\leqq & M \sum\left|u_{j}(n)-u_{0}(n)\right| h(n) \\
\leqq & M \sum_{n=0}^{K}\left|u_{j}(n)-u_{0}(n)\right| h(n)+M \sum_{n=K+1}^{\infty} u_{0}(n) h(n) \\
& +M \sum_{n=K+1}^{\infty} u_{j}(n) h(n) \\
\leqq & \frac{\varepsilon}{2}+M \sum_{n=K+1}^{\infty} u_{j}(n) h(n) .
\end{aligned}
$$

Now

$$
\begin{aligned}
M \sum_{n=K+1}^{\infty} u_{j}(n) h(n) & \leqq M \sum_{n=K+1}^{\infty} u_{0}(n) h(n)+M\left|\sum_{n=K+1}^{\infty}\left[u_{j}(n)-u_{0}(n)\right] h(n)\right| \\
& \leqq \frac{\varepsilon}{4}+M\left|\sum_{n=0}^{K}\left[u_{j}(n)-u_{0}(n)\right] h(n)\right| \leqq \varepsilon / 2 .
\end{aligned}
$$

Thus $\left|f_{j}(x)-f_{0}(x)\right| \leqq \varepsilon$, if $j \geqq J$.

To prove the converse, let $\left\{\left\langle u_{j}\right\rangle\right\}_{j \in N^{\prime}}$ be distribution sequences, and let $\left\{f_{j}\right\}_{j \in N^{\prime}}$ be their respective characteristic functions. Assume that for each $x \in I, f_{j}(x) \rightarrow f(x)$ as $j \rightarrow \infty$, where $f$ is a function which is continuous at $b$. Since $f_{j}(b)=1\left(j \in N^{\prime}\right)$, it follows that

$$
f(t) \longrightarrow f(b)=1 \text { as } t \longrightarrow b \text {. }
$$

Let $u(n)=\int f \bar{\phi}_{n} d \alpha$, then by Lebesgue's dominated convergence theorem $u_{j}(n)=\int f_{j} \bar{\phi}_{n} d \alpha \rightarrow \int f \bar{\phi}_{n} d \alpha=u(n)$ as $j \rightarrow \infty$, so $u(n) \geqq 0$; and for any $K$,

$$
\sum_{n=0}^{K} u(n) h(n)=\lim _{j \rightarrow \infty} \sum_{n=0}^{K} u_{j}(n) h(n) \leqq 1,
$$

so $\sum u(n) h(n) \leqq 1$. From Lemma 1, it follows that

$$
f=\sum u(n) h(n) \dot{\phi}_{n}
$$

except on a null set with respect to $d \alpha$, so

$$
\lim _{x \rightarrow b} \sum u(n) h(n) \dot{\phi}_{n}(x)=\lim _{x \rightarrow b} f(x)=1 .
$$

By Lebesgue's dominated convergence theorem and Axiom A3, it follows that $\sum u(n) h(n)=1$; so $\langle u\rangle$ is a distribution sequence. Now the first part of (c) tells us that the sequence $f_{j}$ converges to its limit uniformly, so $f$ is continuous, and (6) holds everywhere on $I$, thus $f$ 
is a characteristic function.

The following corollary justifies the replacement of $M$ by 1 in A4.

COROLlaRy. $\left|\dot{\phi}_{n}(x)\right| \leqq 1$ for $x \in I$.

Proof. By Theorem $1(\mathrm{~b})\left(\phi_{n}\right)^{p}$ is a characteristic function for each $p \in N^{\prime}$. Let $a^{(p)}(k)=\int\left(\dot{\phi}_{n}\right)^{p} \bar{\phi}_{k} d \alpha$, then $\sum h(k) a^{(p)}(k)=1$, so

$$
\left|\dot{\phi}_{n}(x)\right|^{p}=\left|\sum a^{(p)}(k) h(k) \phi_{k}(x)\right| \leqq \sum \alpha^{(p)}(k) h(k) M \leqq M ;
$$

i.e.,

$$
\left|\dot{\phi}_{n}(x)\right|^{p} \leqq M \quad\left(p \in N^{\prime}\right)
$$

so $\left|\dot{\phi}_{n}(x)\right| \leqq 1$.

A class of characteristic functions which play a particularly important role in our investigation are the divisible characteristic functions: we say a characteristic function $f$ is divisible if for every $n \in N^{\prime}$, there is a characteristic function $f_{n}$ such that $\left(f_{n}\right)^{n}=f$. Note that this definition is identical to the classical one (the phrase "infinitely divisible" is often used). $f_{n}$ can be taken to be the continuous $n$th root of $f$ which takes the value 1 when $t=b$, and is denoted by $f^{1 / n}$. The following theorem lists the basic properties of divisible characteristic functions.

THEOREM 2. (a) Divisible characteristic functions have no zeros on $[a, b]$.

(b) Products of divisible characteristic functions are divisible.

(c) If $\left\{f_{j}\right\}_{j \in N^{\prime}}$ is a sequence of divisible characteristic functions that converges to a characteristic function $f$, then $f$ is divisible.

(d) If $f$ is a divisible characteristic function, then $f^{p}$ is a divisible characteristic function for each $p>0$.

(e) If $g$ is a characteristic function and if $p>0$, then $f=$ $\exp \{p(g-1)\}$ is a divisible characteristic function.

(f) Suppose $u(n) \geqq 0\left(n \in N^{\prime}\right)$ and $\Sigma^{\prime} u(n) h(n)<\infty$, then

$$
f=\exp \left[\Sigma^{\prime} u(n) \bar{h}(n)\left(\dot{\phi}_{n}-1\right)\right]
$$

is an infinitely divisible characteristic function; conversely every infinitely divisible characteristic function can be uniquely expressed in the form (7).

The formula (7) is analogous to the Lévy-Khinchine canonical representation of divisible characteristic functions.

Proof. (a)-(e) are similar in statement and proof with theorems that can be found in [12, pp. 108-113]. 
Proof of (f). If $u(n) \geqq 0$ and $A=\sum^{\prime} u(n) h(n)<\infty$, then $f=$ $\exp \left\{\sum^{\prime} u(n) h(n)\left(\phi_{n}-1\right)\right\}=\exp \left\{A\left[\left(\sum^{\prime} A^{-1} u(n) h(n) \phi_{n}\right)-1\right]\right\}$ is a divisible characteristic function by (e). To demonstrate the converse, let $f$ be a divisible characteristic function, then for each $m \in N^{\prime}$

$$
f^{1 / m}=\sum u_{m}(n) h(n) \phi_{n}
$$

with $u_{m}(n) \geqq 0\left(m \in N^{\prime}, n \in N\right)$ and $\sum u_{m}(n) h(n)=1$. Now

$$
g_{m}=m\left(f^{1 / m}-1\right)=m \sum^{\prime} u_{m}(n) h(n)\left[\phi_{n}-1\right],
$$

so $f_{m}=\exp \left(g_{m}\right)$ is a characteristic function by (e) and $f_{m}$ converges pointwise, indeed uniformly by Theorem 1 (c) to $f$. Since $f$ is divisible, it is bounded away from zero, whence $g_{m} \rightarrow g=\log f$ uniformly. Thus for $n \in N^{\prime} m u_{m}(n)=\int g_{m} \phi_{n} d \alpha$ converges to $\int g \phi_{n} d \alpha \equiv u(n)$, hence $u(n) \geqq$ 0. Now

$$
m \Sigma^{\prime} u_{m}(n) h(n)=-\left(\int d \alpha\right)^{-1} \int g_{m} d \alpha \longrightarrow-\left(\int d \alpha\right)^{-1} \int g d \alpha=B,
$$

whence $m \sum^{\prime} u_{m}(n) h(n) \leqq B+1$ for $m$ sufficiently large, and thus $\Sigma^{\prime} u(n) h(n) \leqq B+1$, so (f) is proved.

We say a distribution sequence $\langle u\rangle$ is divisible if for each $n$ there is a distribution sequence $\left\langle u_{n}\right\rangle$ such that $\left\langle u_{n}^{* n}\right\rangle=\langle u\rangle$ where, $\left\langle u^{* 1}\right\rangle=\langle u\rangle$ and $\left\langle u^{* n}\right\rangle=\left\langle u * u^{*(n-1)}\right\rangle$. Then an immediate consequence of $(f)$ is the

CoRollary. A distribution sequence $\langle u\rangle$ is divisible if and only if $\langle u\rangle$ has the form

$$
\langle u\rangle=\left\langle e^{\left.-\lambda\left[\sum_{n=0}^{\infty} \frac{\lambda^{n}}{n !} v^{* n}\right]\right\rangle}\right.
$$

for some distribution sequence $\langle v\rangle$.

REMARKS. 1. If $f$ is divisible, then $(f)^{1 / m}$ is a characteristic function which converges to the constant function 1 as $m \rightarrow \infty$, thus the existence of divisible functions implies Axiom A2. Conversely Axiom A2 states that 1 is a characteristic function; 1 is obviously divisible, so A2 is equivalent to the existence of divisible functions; or, what is the same, the existence of divisible functions is equivalent to 1 being a characteristic function.

2. An alternate approach to this subject is to take the convolution algebra as the primitive notion. This is the method used by Gilewski and Urbanik [8]. Let $\mathscr{P}$ denote the set of nonnegative measures on $N$ with total mass not exceeding 1 , and $\mathscr{P}_{0}$ be the probability measures in $\mathscr{P}$. We say $P_{k} \rightarrow P$ if $P_{k}(n) \rightarrow P(n)$ for each $n \in N$. $E_{k}$ stands for the unit mass concentrated at $k$. A binary operation ${ }^{*}$ on 
$\mathscr{P}$ is a generalized convolution if it satisfies

(i) $E_{0} * P=P\left(P \in \mathscr{P}^{\circ}\right)$.

(ii) $(a P+b Q) * R=a(P * R)+b(Q * R)$

$\left(P, Q, R \in \mathscr{P}_{,}, a, b>0\right.$, and $\left.a+b \leqq 1\right)$.

(iii) If $P_{k} \rightarrow P$, then $P_{k} * Q \rightarrow P * Q,\left(P, P_{k}, Q \in \mathscr{P}\right)$.

(iv) $P * Q \in \mathscr{P}_{0}$ whenever $P, Q \in \mathscr{P}_{0}$.

The convolution is studied by means of a generating function which is a one-to-one mapping from $\mathscr{P}$ into the analytic functions on the unit disc. The image of $P$, called the generating function of $P$, is denoted by $\Phi_{p}$ and must satisfy

(i ) $\Phi_{a P+b Q}=a \Phi_{p}+b \Phi_{Q},(P, Q \in \mathscr{P}$ and, $a, b \geqq 0, a+b \leqq 1)$.

(ii) $\Phi_{P * Q}=\Phi_{P} \Phi_{Q}(P, Q \in \mathscr{P})$.

(iii) $\Phi_{P_{k}} \rightarrow \Phi_{P}$ uniformly on compact subsets of the disk if and only if $P_{k} \rightarrow P$.

In this context, then, Gilewski and Urbanik obtain a result analogous to Theorem 2 (f).

In this general setting a characteristic function can be defined by requiring the $\Phi_{P}$ to be continuous on some interval $[a, b]$ and replacing (iii) by (iii') $\Phi_{P_{k}} \rightarrow \Phi_{P}$ uniformly if and only if $P_{k} \rightarrow P$.

3. Stable characteristic functions. In the classical case, a distribution function $F$ is said to be stable if to every $b_{1}>0, b_{2}>0$, and real $c_{1}, c_{2}$, there corresponds a positive number $b$ and a real number $c$ such that if $F_{i}=F\left(\left(x-c_{i}\right) / b_{i}\right)$, then $F_{1} * F_{2}(x)=F((x-c) / b)$. The corresponding relation for the characteristic functions is that $f\left(b_{1} t\right) f\left(b_{2} t\right)=$ $f(b t) e^{i \alpha t}$ where $\alpha=c-c_{1}-c_{2}$. Thus the study of stable characteristic functions reduces to a study of characteristic functions which have the following property: given positive real numbers $b_{1}$ and $b_{2}$, there exists a positive real number $b$ such that $f\left(b_{1} t\right) f\left(b_{2} t\right)=f(b t)$. A canonical form for all stable distributions in the classical case can be found in [12, p. 132].

The balance of the paper is devoted to the formulation of an appropriate notion of stability and to the identification of the stable characteristic functions as so defined.

In the classical definition of stability, a set of functions was required to exhibit a certain behavior with respect to a semigroup of functions on the line. This notion is generalized so that stability with respect to a semigroup acting on $I$ can be defined.

Reminiscent of the semigroup $s_{c}(x)=c x$ define a dilation to be a 1-parameter family of function $s_{c}$ each one being defined at least on $I$ such that $s_{c}$ is strictly increasing if $c>0$ and the following properties all hold

B1. $s_{0}(x) \equiv b$.

B2. $s_{o}(b)=b(c \geqq 0)$. 
B3. $s_{c}\left(s_{d}(x)\right)$ is defined if $c, d \geqq 0$ and $x \in I$ and $s_{c}\left(s_{d}(x)\right)=s_{c d}(x)$.

B4. $s_{1}(x)=x$.

B5. $s_{c}(x)$ is continuous in $c$ and in $x$.

As examples, consider $s_{c}(x)=b-c(b-x)$, and $s_{c}(x)=(b+1)-$ $(b+1-x)^{c}$.

A definition of stability can now be given. Let $s$ be a dilation and let $E(s)=\left\{s_{c}(x) \mid c \geqq 0, x \in I\right\}$; write $g_{c}(x)$ for $g\left(s_{c}(x)\right)$ if there is no danger of confusion. A function $f$ defined on $E(s)$ is said to be stable with respect to $s$ (or simply stable if there is no ambiguity) if $f_{c}$ restricted to $I$ is a characteristic function for each $c \geqq 0$, and if corresponding to each $c \geqq 0$ and $d \geqq 0$ there is an $e \geqq 0$ such that $f_{c}(x) f_{d}(x)=f_{e}(x)$ for $x \in I$; when this is the case write

$$
c \circ d=e
$$

If $f$ is stable with respect to $s$ say $(f, s)$ is a stable pair. As a trivial example, if $f$ is the function which is equal to one at each point of $I$, then $(f, s)$ is a stable pair for every dilation $s$.

In order to state the main result a canonical form for dilations is necessary. This is given in Lemma 3, below, the proof of which requires

Lemma 2. (a) For each $x \in I, x \neq b, s_{c}(x)$ is a strictly decreasing function of $c$.

(b) For each $x \in I, x \neq b,\left\{s_{c}(x) \mid c \geqq 0\right\} \supseteqq I$.

Proof. Since $s_{c}(x)$ is continuous in $c$ and $s_{1}(x)=x<b=s_{0}(x)$ it will suffice to show that if $c \neq d, s_{c}(x) \neq s_{d}(x)$. Suppose by way of contradiction that $s_{c}(x)=s_{d}(x)$, and assume that $\lambda=d / c<1$. Then application of $s_{1 / c}$ to both sides of the last equation yields $x=s_{\lambda}(x)$; repeated application of $s_{\lambda}$ leads to $x=s_{\lambda, n}(x)$. But, $\lambda_{n} \rightarrow 0$, so $s \lambda^{n}(x) \rightarrow b$, hence $x=b$. This contradiction establishes (a).

To prove (b) let $y \in I$. If $y \geqq x$ then $s_{1}(x)=x \leqq y \leqq b=s_{0}(x)$ so $y=s_{c}(x)$ for some $c \leqq 1$. If $y<x$ then $x=s_{d}(y)$ for some $d$ in $(0,1]$, but then $y=s_{1 / d}(x)$, so (b) is proved.

LEMma 3. Let is be a strictly decreasing continuous function defined on an interval containing I and with range containing $[0, \infty)$ such that $\psi(b)=0$. Then if we define

$$
s_{c}(x)=\psi^{-1}(c(\psi(x))) \quad(c \geqq 0)
$$

$s$ is a dilation; conversely if $s$ is a dilation there is a strictly decreasing continuous function is defined on an interval containing $I$ and with range containing $[0, \infty)$ such that $\psi(b)=0$ satisfying (8). 
Proof. If $\psi$ satisfies the hypothesis then B1-B4 follow by direct computation and B5 is trivial.

To prove the converse, let $x_{0} \in I, x_{0} \neq b$ and define $\psi$ by $\psi^{-1}(t)=$ $s_{t}\left(x_{0}\right) . \quad \psi$ is well defined by Lemma $2(a)$. The domain and range of $\psi$ are correct by Lemma $2(b)$. Finally, to establish (8) let $x \in I$. Then for some $d \geqq 0, x=s_{d}\left(x_{0}\right)$, so $\psi^{-1}(c(\psi(x)))=\psi^{-1}\left(c\left(\psi\left(s_{d}\left(x_{0}\right)\right)\right)\right)=$ $\psi^{-1}(c d)=s_{c d}\left(x_{0}\right)=s_{c}\left(s_{d}\left(x_{0}\right)\right)=s_{c}(x)$.

4. The main result.

THEOREM 3. Let $s$ be a dilation determined by the function $\psi$ as in (8) and let $B=\sup \left\{\beta \mid 1-\lambda \psi^{\beta}\right.$ is a characteristic function for some $\lambda>0\}$,

(a) There are nontrivial stable pairs $(f, s)$ if and only if $B>0$.

(b) If $B>0,(f, s)$ is a stable pair if and only if

$$
f=\exp \left[-m \psi^{\beta}\right] \text { for some } m \geqq 0 \text { and } 0 \leqq \beta \leqq B \text {. }
$$

REMARKS. The hypothesis of (a) can be more simply stated: for some $\lambda>0$ and $\beta>0,1-\lambda \psi^{\beta}$ is a characteristic function.

The proof of the theorem will follow from a number of lemmas. In the following assume $(f, s)$ is a fixed nontrivial stable pair and that $s$ is determined by a function $\psi$ as in (8).

Define $c(0)=0$ and $c(n)=1 \circ c(n-1) \quad\left(n \in N^{\prime}\right)$. The following lemma describes the behavior of $f_{c}$ and $c(n)$.

LEMMA 4. (a) $f_{1}=f, f_{0}=1,\left(f_{a}\right)_{b}=f_{a b}$, and $f_{a} f_{b}=f_{a \circ b}$.

(b) $f_{c(n)}=f^{n}\left(n \in N^{\prime}\right)$.

(c) $c(n) \neq 0\left(n \in N^{\prime}\right)$.

(d) $f$ is divisible.

(e) If $x \neq y$ then $f_{x} \neq f_{y}$.

Proof. (a) follows by trivial computations, and (b) follows by iterating the last relation in (a), (c) follows from (b) and the assumption that $f$ is not constant.

Since $f$ is stable $f_{1 / o(n)}$ is a characteristic function for $n \in N^{\prime}$. Moreover $\left[f_{1 / c(n)}\right]^{n}=\left[f^{n}\right]_{1 / c(n)}=\left[f_{c(n)}\right]_{1 / c(n)}=f_{1}=f$, so (d) is proved. To show (e), assume by way of contradiction that $x>y$; but $f_{x}=f_{y}$ so we have $\left(f_{x}\right)_{1 / x}=\left(f_{y}\right)_{1 / x}$ or $f=f_{y / x}$. Thus $f=f_{(y / x)^{k}}\left(k \in N^{\prime}\right)$, but $(y / x)^{k} \rightarrow 0$ as $k \rightarrow \infty$ and $f$ and $s$ are continuous, so $f \equiv 1$ which is a contradiction.

The next task is to extend $c$ to a function on $[0, \infty)$ which satisfies

$$
f_{c(x)}=f^{x} \text {. }
$$

Since $f$ is divisible, $f^{x}$ is a characteristic function for every positive 
$x$, so by (e) of Lemma 4 there is at most one positive number $c(x)$ such that (10) holds. Let $X$ denote the set of all $x \geqq 0$ for which such a $c(x)$ exists.

LEMMA 5. $X$ is closed under multiplication and division; if $x \in X$ and $y \in X$, then $c(x y)=c(x) c(y)$. Moreover, $X$ is dense in $[0, \infty)$.

Proof. If $x$ and $y$ are in $X, f^{x y}=\left[f_{c(x)}\right]^{y}=f_{c(x) c(y)}$, so $x y \in X$ and by (e) of Lemma $4 c(x y)=c(x) c(y)$. If $x \in X, x \neq 0, f=\left[f_{c(x)}\right]_{1 / c(x)}=$ $\left[f^{x}\right]_{1 / c(x)}=\left[f_{1 / c(x)}\right]^{x}$, so $f^{1 / x}=f_{1 / c(x)}$, whence $1 / x \in X$ and $c(1 / x)=1 / c(x)$. Thus $X$ is closed under division, the last assertion follows because $X$ contains the positive rational numbers.

There is now enough information to obtain the exact form of $c$ :

Lemma 6. $X=[0, \infty)$ and $c(x)=x^{\gamma}$ for some $\gamma>0$, so that

$$
f^{x}=f_{x r} \text {. }
$$

Proof. Let $x \in[0, \infty)$, and let $\{x(n)\}_{n \in-}$ be a sequence in $X$ which converges to $x$. By taking a subsequence, it may be assumed that $c(x(n)) \rightarrow c_{0} \in[0, \infty]$ as $n \rightarrow \infty$. If $c_{0}=\infty$, let $z(n)=1 / x(n)$, then $z(n) \in X(n \in N)$ and $c(z(n)) \rightarrow 0$ as $n \rightarrow \infty$. Thus $f^{1 / x}=\lim _{n \rightarrow \infty} f^{z(n)}=$ $\lim _{n \rightarrow \infty} f_{c(z(n))}=f_{0}$, or $f^{1 / x}=f_{0}$, and by Lemma 4, this is not possible for any real $x$; hence $c_{0}<\infty$.

In this case, $f_{c_{0}}=\lim _{n \rightarrow \infty} f_{c(x(n))}=\lim _{n \rightarrow \infty} f^{x(n)}=f^{x}$, or $f^{x}=f_{c_{0}}$, thus $x \in X$ and $c(x)=\varepsilon_{0}$. Moreover, $c$ is continuous at $x$ since Lemma $4 \mathrm{e}$ shows that if $x(n)$ is any sequence which converges to $x$ then $\underline{\lim } c(x(n))=\varlimsup \lim c(x(n))=c(x)$, so $\lim c(x(n))=c(x)$.

Thus $c(x y)=c(x) c(y)$ for positive reals $x$ and $y, c(0)=0$, and $c$ is continuous. These conditions guarantee that $c(x)=x^{i}$ for some $\gamma \geqq 0$. This can be seen by considering the function $L(t)=\log c\left(e^{t}\right)$ for $t$ real. $L$ is continuous and linear so $L(t)=\gamma t$ for some real $\gamma$, whence $c(x)=$ $x^{r}$ for some real $\gamma$; finally $\gamma>0$ since $c(0)=0$.

The question is now: for which $\beta$ does (9) describes a characteristic function. A partial answer is given by the following lemma which adopts Bochner's technique of subordination (cf. [4, pp. 91-93]) to the present case.

LEMIIA 7. If for some function $g$, exp (-mg) is a characteristic function for each $m \geqq 0$ then so is exp $\left(-m g^{3}\right)$ for each $m \geqq 0$ and $0<\beta<1$.

Proof. First observe that exp $\left(-m x^{\beta}\right)$ is completely monotone for $x>0$; i.e., its derivatives alternate in sign. Thus by Bernstein's theorem [15, p. 161], it is the Laplace-Stieltjes transform of a positive 
measure $\rho$ :

$$
\exp \left(-m x^{\beta}\right)=\int_{0}^{\infty} \exp (-x t) d \rho(t) \quad(x>0) .
$$

$\rho$ has, in fact, total variation one as can be seen by letting $x$ decrease to zero and using Lebesgue's monotone convergence theorem.

Now define $t(n, i)=i / n$ for $i=0,1, \cdots, n^{2}$ and $n \in N^{\prime}$, and set $E(n, i)=[t(n, i), t(n, i+1)]$ if $i=0,1, \cdots, n^{2}-1$, and $E\left(n, n^{2}\right)=$ $[n, \infty)$.

$$
f_{n}(x)=\sum_{i=0}^{n^{2}} \exp \{-t(n, i) g(x)\} \rho[E(n, i)]
$$

is a characteristic function because it is a convex combination of characteristic functions. On the other hand, it is elementary to show that

$$
\lim _{n \rightarrow \infty} f_{n}(x)=\int \exp (-t g(x)) d \rho(t)=\exp \left\{-m[g(x)]^{\beta}\right\} .
$$

So $\exp \left\{-m[g(x)]^{\beta}\right\}$ is a pointwise limit of characteristic functions. Since it is continuous, it is itself a characteristic function by Theorem 1 (c).

Proof of Theorem 3. Let $s, \psi$, and $B$ be as in the statement of the theorem. Define

$$
f^{(m, \beta)}=\exp \left\{-m \psi^{\beta}\right\} .
$$

The set of all stable functions is

$$
S=\left\{f^{(m, \beta)} \mid m \geqq 0 \text { and } 0 \leqq \beta \leqq B\right\} .
$$

To see this, let $f=f^{(m, \beta)} \in S$ and assume $0<\beta<B$. Then for some positive $\lambda, 1-\lambda \psi^{\beta}$ is a characteristic function; so by Theorem $2(\mathrm{e})$, $f^{(m, \beta)}$ is also, as long as $m>0$. Moreover

$$
f_{c}=\exp \left\{-m c^{\beta} \psi^{\beta}\right\}
$$

so that $f_{c}$ is a characteristic function for every $c \geqq 0$, it also follows that

$$
f_{c} f_{d}=f_{e} \quad \text { where } e^{\beta}=c^{\beta}+d^{\beta},
$$

so $f$ is stable.

To show $f^{(m, B)}$ is stable, let $\beta$ increase to $B$, then $f_{c}^{(m, \beta)}$ converges pointwise to $f_{c}^{(m, B)}$ which is continuous at $b$, so by Theorem 1 (c) $f_{c}^{(m, B)}$ is a characteristic function for every $c \geqq 0$. Now fix $c$ and $d$, and let

$$
e(\beta)=\left(c^{\beta}+d^{\beta}\right)^{1 / \beta} ;
$$


so, if $0<\beta<B$, it follows from (12) that

$$
f_{c}^{(m, \beta)} f_{d}^{(m, \beta)}=f_{e(\beta)}^{(m, \beta)} \text {. }
$$

Again, letting $\beta$ increase to $B$ it is found that $f^{(m, B)}$ is stable. Thus the set $S$ consists entirely of stable functions.

Now suppose $f$ is stable, by Lemma 6 there is $\gamma>0$ so that (11) holds. Let $g=f^{(m, 1 / r)}$ then a direct computation shows

$$
g^{x}=g_{x}
$$

(even if $1 / \gamma>B$ ). Using (11) and (13), if

$$
\begin{aligned}
k & =\log f / \log g \\
k\left(s_{x \gamma}(t)\right) & =\frac{\log f\left(s_{x \gamma}(t)\right)}{\log g\left(s_{x \gamma}(t)\right)}=\frac{x \log f(t)}{x \log g(t)}=k(t) .
\end{aligned}
$$

If $t_{1} \neq t$, by Lemma $2(\mathrm{~b})$ an $x$ can be found such that $t_{1}=s_{x r}(t)$, hence $k\left(t_{1}\right)=k\left(s_{x \gamma}(t)\right)=k(t)$ and $k$ must then be constant so

$$
f=f^{(M, \beta)}=\exp \left\{-M \psi^{\beta}\right\}
$$

for some constant $M$ and $\beta=1 / \gamma$.

Since $f$ is a stable characteristic function, it is divisible by Lemma 4 (d), so by Theorem 2 (f) there must be $a(n) \geqq 0 n \in N^{\prime}$ such that $\Sigma^{\prime} a(n) h(n)=A<\infty$

$$
f=\exp \left[\Sigma^{\prime} a(n) h(n)\left(\phi_{n}-1\right)\right] .
$$

Comparing (14) and (15),

$$
-M \psi^{\beta}=\Sigma^{\prime} a(n) h(n)\left(\phi_{n}-1\right),
$$

or

$$
1-\lambda \psi^{\beta}=\sum_{n=1}^{\infty} A^{-1} a(n) h(n) \phi_{n}
$$

where $\lambda=M / A$. The right hand side of (16) is a characteristic function, hence $0<\beta \leqq B . \quad M>0$ because $f$ is a characteristic function so $f \in S$, and $S$ is exactly the set of stable functions, and part (b) of the theorem is proved.

In the course of the above argument stable functions were produced under the assumption $B>0$, and it was shown that if $f$ was nontrivial and stable, $f=f^{(m, \beta)}$ for some $\beta>0$, which implies $B>0$, thus (a) is proved also.

5. Examples. Now a detailed analysis of the simplest $I$-semigroup is made in the following 
THEOREM 4. If $\left\{\phi_{n}\right\}_{n \in N}$ are polynomials and $s_{c}(x)=b-c(b-x)$, then the characteristic functions which are stable with respect to $S$ have the form

$$
\exp \left\{-m(b-x)^{\beta}\right\} \quad(m \geqq 0,0<\beta \leqq 1) .
$$

The proof, which appears after the following lemma, consists of two parts: the first being to show that $f(x)=1-\lambda(b-x)$ is a characteristic function for some $\lambda>0$, which implies by Theorem 3 that functions of the form (17) are stable, and the second part will show that $\beta$ cannot exceed 1.

The following lemma is used in both parts of the proof.

LEMMA 8. $\phi_{n}^{\prime}(b)>0$ if $n>1$.

Proof. The zeros of $\phi_{n}$ are simple and contained in $(a, b)[14$, p. 44]; by Rolle's theorem, the same holds for $\phi_{n}^{\prime}$. Thus $\phi_{n}^{\prime}$ has constant, nonzero sign on $[b, \infty)$. Since $\phi_{n}$ is a polynomial and since $\phi_{n}(b)=1$, the lemma follows.

Proof of Theorem 4. Define the moments $\mu(i)=\int x^{i} d \alpha(x) . \quad \phi_{1}$ is a first degree polynomial satisfying $\phi_{1}(b)=1$, so $\phi_{1}(x)=1-c(b-x)$. Lemma 8 implies that

$$
c>0 \text {. }
$$

$f$ is also a polynomial of degree one so

$$
f(x)=1-\lambda(b-x)=a(0) h(0)+a(1) h(1) \dot{\phi}_{1}(x) .
$$

Differentiation of the above yields

$$
\lambda=a(1) h(1) c .
$$

Since $f$ is bounded by 1 on $I, \lambda>0$, so (18) implies

$$
a(1)>0 \text {. }
$$

Also $a(0)=\int f d \alpha=1-\lambda(b \mu(0)-\mu(1))$. Thus for small enough positive $\lambda$

$$
a(0)>0 \text {. }
$$

Evaluation of (19) at $x=b$ shows

$$
a(0) h(0)+a(1) h(1)=1,
$$

and so (20), (21), and (22) imply that $f$ is a characteristic function for 
sufficiently small $\lambda$.

To see that $\beta \leqq 1$, note that if $f=\sum a(n) h(n) \phi_{n}$ is a characteristic function which is differentiable at $b$, then

$$
\sum a(n) h(n) \phi_{n}^{\prime}(b) \leqq f^{\prime}(b)
$$

(this is readily shown by Fatou's lemma). In particular, Lemma 8 guarantees that if $f$ is differentiable at $b$,

$$
f^{\prime}(b)>0
$$

Thus if $f(t)=\exp \left\{-m(b-t)^{\beta}\right\}$, then $\beta$ cannot exceed 1 without violating (23).

It is easy to see the conditions on $s$ and $\left\{\phi_{n}\right\}$ which make Theorem 4 possible. They are stated in the following theorem which is given without proof.

THEOREM 5. Suppose either

(a) $\left\{\phi_{n}\right\}$ are real orthogonal polynomials, or

(b) $\dot{\phi}_{0} \equiv 1$, and for some complex number $\alpha$ and all $n \in N^{\prime}$

$$
\liminf _{x \rightarrow b-} \alpha \frac{1-\phi_{n}(x)}{b-x}>0 \text {. }
$$

Let $s, \psi$, and $B$ be as in Theorem 3 , and assume $1-\lambda \psi$ is a characteristic function for some $\lambda>0$, then the set of functions stable with respect to $s$ is

$$
\left\{\exp \left\{-m \psi^{\beta}\right\} \mid m \geqq 0,0<\beta \leqq 1\right\} \text {. }
$$

\section{REFERENCES}

1. N. H. Bingham, Random walks on spheres, Z. Wahrscheinlichkeitstheorie Verw. Geb., 22 (1972), 169-192.

2. - Positive definite functions on spheres, Proc. Camb. Philos. Soc., 73 (1973), $145-156$.

3. S. Bochner, Positive zonal functions on spheres, Proc. Nat. Acad. Sci., U.S.A., 40 (1954), 1141-1147.

4. - Harmonic Analysis and the Theory of Probability, University of California Press, Berkeley, (1960).

5. S. D. Conte, Gegenbauer Transforms, Quart. J. Math., (Oxford) (2) 6 (1955), 48-52. 6. G. Gasper, Linearization of the product of Jacobi polynomials, I. Canad. J. Math., XXII (1970), 171-175.

7. - Linearization of the product of Jacobi polynomials, II, Canad. J. Math., XXII (1970), 582-593.

8. J. Gilewski and K. Urbanik, Generalized convolutions and generating functions, Bull. Acad. Sci. Polon. Ser. Math. Astr. Phys., 16 (1968), 481-487.

9. I. I. Hirschman, Jr., Sur les polynomes ultrasphériques, Comptes Rendus, 242 (1956), 2212-2214.

10. M. Kennedy, A stochastic process associated with the ultraspherical polynomials, Proc. Roy. Irish Acad., 61 (1961), 89-100. 
11. J. Lamperti, The arithmetic of certain semigroups of positive operators, Proc. Camb. Phil. Soc., 64 (1968), 161-166.

12. E. Lukacs, Characteristic Functions, Hafner Publishing Co., New York, 1970.

13. P. H. Roberts and H. D. Ursell, Random walk on a sphere and on a Riemannian manifold, Phil. Trans. Roy. Soc. London, Ser. A, 252 (1960), 317-356.

14. G. Szegö, Orthogonal Polynomials, Amer. Math. Soc. Colloq. Pub., Vol. 23, American Mathematical Society, Providence, 1967.

15. D. Widder, The Laplace Transform, Princeton University Press, Princeton, 1946.

Received March 12, 1973 and in revised form March 13, 1974. The work in this paper was supported by the University of Missouri, St. Louis and the Air Force Office of Scientific Research Grant number 71-2047.

UNIVERSITY OF Missouri-St. LOUIS 


\section{PACIFIC JOURNAL OF MATHEMATICS}

\section{EDITORS}

RICHARD ARENS (Managing Editor)

University of California

Los Angeles, California 90024

\section{R. A. Beaumont \\ University of Washington \\ Seattle, Washington 98105}

\section{J. DugundJI}

Department of Mathematics

University of Southern California

Los Angeles, California 90007

D. Gilbarg and J. Milgram

Stanford University

Stanford, California 94305

\section{ASSOCIATE EDITORS}
E. F. BECKENBACH
B. H. NeumanN
F. WOLF
K. YOSHIDA

\section{SUPPORTING INSTITUTIONS}

UNIVERSITY OF BRITISH COLUMBIA
CALIFORNIA INSTITUTE OF TECHNOLOGY
UNIVERSITY OF CALIFORNIA
MONTANA STATE UNIVERSITY
UNIVERSITY OF NEVADA
NEW MEXICO STATE UNIVERSITY
OREGON STATE UNIVERSITY
UNIVERSITY OF OREGON
OSAKA UNIVERSITY

UNIVERSITY OF BRITISH COLUMBIA

UNIVERSITY OF CALIFORNIA

MONTANA STATE UNIVERSITY

NEW MEXICO STATE UNIVERSITY

OREGON STATE UNIVERSITY

OSAKA UNIVERSITY

\author{
UNIVERSITY OF SOUTHERN CALIFORNIA \\ STANFORD UNIVERSITY \\ UNIVERSITY OF TOKYO \\ UNIVERSITY OF UTAH \\ WASHINGTON STATE UNIVERSITY \\ UNIVERSITY OF WASHINGTON \\ AMERICAN MATHEMATICAL SOCIETY \\ NAVAL WEAPONS CENTER
}

The Supporting Institutions listed above contribute to the cost of publication of this Journal, but they are not owners or publishers and have no responsibility for its content or policies.

Mathematical papers intended for publication in the Pacific Journal of Mathematics should be in typed form or offset-reproduced, (not dittoed), double spaced with large margins. Underline Greek letters in red, German in green, and script in blue. The first paragraph or two must be capable of being used separately as a synopsis of the entire paper. Items of the bibliography should not be cited there unless absolutely necessary, in which case they must be identified by author and Journal, rather than by item number. Manuscripts, in triplicate, may be sent to any one of the editors. Please classify according to the scheme of Math. Reviews, Index to Vol. 39. All other communications should be addressed to the managing editor, or Elaine Barth, University of California, Los Angeles, California, 90024.

The Pacific Journal of Mathematics expects the author's institution to pay page charges, and reserves the right to delay publication for nonpayment of charges in case of financial emergency.

100 reprints are provided free for each article, only if page charges have been substantially paid. Additional copies may be obtained at cost in multiples of 50 .

The Pacific Journal of Mathematics is issued monthly as of January 1966. Regular subscription rate: $\$ 72.00$ a year (6 Vols., 12 issues). Special rate: $\$ 36.00$ a year to individual members of supporting institutions.

Subscriptions, orders for back numbers, and changes of address should be sent to Pacific Journal of Mathematics, 103 Highland Boulevard, Berkeley, California, 94708.

PUBLISHED BY PACIFIC JOURNAL OF MATHEMATICS, A NON-PROFIT CORPORATION

Printed at Kokusai Bunken Insatsusha (International Academic Printing Co., Ltd.), 270, 3-chome Totsuka-cho, Shinjuku-ku, Tokyo 160, Japan.

Copyright (C) 1973 by Pacific Journal of Mathematics Manufactured and first issued in Japan 


\section{Pacific Journal of Mathematics \\ Vol. 55, No. $2 \quad$ October, 1974}

Walter Allegretto, On the equivalence of two types of oscillation for elliptic

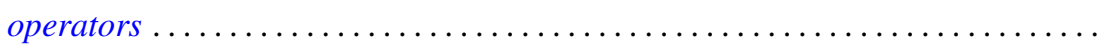

Edward Arthur Bertram, A density theorem on the number of conjugacy classes in

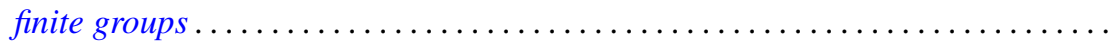

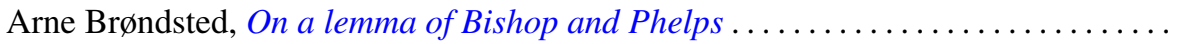

Jacob Burbea, Total positivity and reproducing kernels ..................

Ed Dubinsky, Linear Pincherle sequences . . . . . . . . . . . . . . . . . .

Benny Dan Evans, Cyclic amalgamations of residually finite groups .............

361

Barry J. Gardner and Patrick Noble Stewart, A "going down" theorem for certain

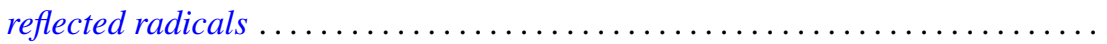

381

Jonathan Light Gross and Thomas William Tucker, Quotients of complete graphs:

revisiting the Heawood map-coloring problem ....................

Sav Roman Harasymiv, Groups of matrices acting on distribution spaces .........

Robert Winship Heath and David John Lutzer, Dugundji extension theorems for

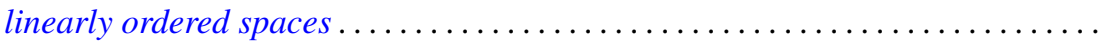

Chung-Wu Ho, Deforming p. l. homeomorphisms on a convex polygonal

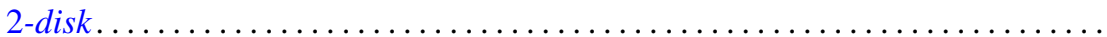

Richard Earl Hodel, Metrizability of topological spaces .................

Wilfried Imrich and Mark E. Watkins, On graphical regular representations of

cyclic extensions of groups .......................... 461

Jozef Krasinkiewicz, Remark on mappings not raising dimension of curves ..... . 479

Melven Robert Krom, Infinite games and special Baire space extensions . . . . . . 483

S. Leela, Stability of measure differential equations . . . . . . . . . . . . . . . . 489

M. H. Lim, Linear transformations on symmetric spaces . . . . . . . . . . . . . . . 499

Teng-Sun Liu, Arnoud C. M. van Rooij and Ju-Kwei Wang, On some group algebra modules related to Wiener's algebra $M_{1} \ldots \ldots \ldots \ldots \ldots \ldots \ldots \ldots \ldots \ldots \ldots$

Dale Wayne Myers, The back-and-forth isomorphism construction ............ 521

Donovan Harold Van Osdol, Extensions of sheaves of commutative algebras by

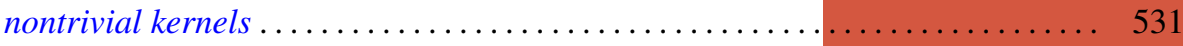

Alan Rahilly, Generalized Hall planes of even order ................... 543

Joylyn Newberry Reed, On completeness and semicompleteness of first countable

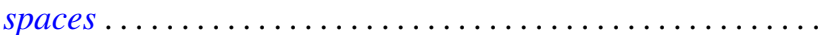

Alan Schwartz, Generalized convolutions and positive definite functions associated

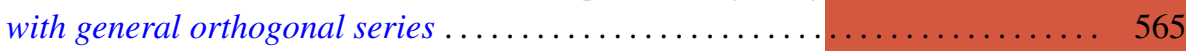

Thomas Jerome Scott, Monotonic permutations of chains . . . . . . . . . . . 583

Eivind Stensholt, An application of Steinberg's construction of twisted groups .... 595

Yasuji Takeuchi, On strongly radicial extensions . . . . ................. 619

William P. Ziemer, Some remarks on harmonic measure in space . . . . . . . . . . 629

John Grant, Corrections to: “Automorphisms definable by formulas” . . . . . . . . 639

Peter Michael Rosenthal, Corrections to: "On an inversion for the general

Mehler-Fock transform pair" ......................... 640

Carl Clifton Faith, Corrections to: "When are proper cyclics injective” . . . . . . 640 